\title{
Sedimentación en comunidades arrecifales de Bahías de Huatulco, Oaxaca, México
}

\author{
María R. Granja Fernández ${ }^{1} \&$ Ramón A. López Pérez ${ }^{2}$ \\ 1. Programa de Biología Marina, Universidad del Mar; oxbec_08@hotmail.com \\ 2. Instituto de Recursos. Universidad del Mar. AP. 47, CP 70902, Puerto Ángel, Oax. México; alopez@angel.umar.mx
}

Recibido 19-XI-2007. Corregido 07-V-2008. Aceptado 09-VI-2008.

\begin{abstract}
Sedimentation on reef communities at Bahías de Huatulco, Oaxaca, Mexico. Although coral reef sedimentation is important because it modifies processes like symbioses, reproduction, recruitment and coral growth, Mexican Pacific studies are lacking. On this regard, spatio-temporal variations in sedimentation rate were investigated in six coral reef communities from Oaxaca. During February 2006-January 2007 (excluding April, July and August) two sediment structures, with four sediment traps each, were randomly installed. Sediment traps were replaced with a mean periodicity of 38 days, and the sediments were washed, filtered, dried and weighted in order to calculate sedimentation rate. Sedimentation rate was heterogeneous among localities $\left(F_{5,36}=7.06, P<0.01\right)$. It was high at Isla Montosa $\left(653.31 \mathrm{~kg} \mathrm{~m}^{-2}\right.$ year $\left.^{-1}\right)$ and Isla Cacaluta $\left(450.09 \mathrm{~kg} \mathrm{~m}^{-2}\right.$ year $\left.{ }^{-1}\right)$, intermediate at San Agustín, Jicaral-Chachacual and Dos Hermanas (155.18-92.53 kg m-2 year $\left.{ }^{-1}\right)$ and low at La Entrega (14.33 $\mathrm{kg} \mathrm{m}^{-2}$ year $\left.^{-1}\right)$. Sedimentation rate was homogeneous through time $\left(F_{7,34}=0.85, P>0.5\right)$; nonetheless, during the dry season (November-March) sedimentation rate in the area oscillated between 6.8-73.5 $\mathrm{mg} \mathrm{cm}^{-2}$ day $^{-1}$, whereas during the rainy season (May-October) the values were 141-1088 \% higher (74.5-147.6 $\mathrm{mg} \mathrm{cm}{ }^{-2}$ day $^{-1}$, Mann-Whitney $\mathrm{U}=137, \mathrm{n}=42, \mathrm{P}=0.03$ ). There was a significant relationship between sedimentation rate and pluvial precipitation (Spearman $R=0.83, n=8, P=0.009$ ), suggesting that the amount of sediment reaching coral communities is closely tied to regional precipitation. Sedimentation rates recorded at Isla Montosa (366.64 mg cm${ }^{-2}$ day $\left.^{-1}\right)$ and Isla Cacaluta (366.03 $\mathrm{mg} \mathrm{cm}^{-2}$ day $\left.^{-1}\right)$ during the rainy season can be considered lethal-sublethal considering sediment tolerance and rejection efficiency of stony corals. The absence of coral mortality during the rainy season may result from: a) high efficiency of active sediment removal, b) increased physiological tolerance to sediments, and c) a high degree of passive sediment removal via turbulence. Nonetheless, the combined effect of natural and anthropogenic induced sedimentation may cause a shift in coral community structure and eventually a loss of the reef areas in Bahías de Huatulco. Rev. Biol. Trop. 56 (3): 11791187. Epub 2008 September 30.
\end{abstract}

Key words: sediments, sedimentation rate, coral communities, Oaxaca, Mexican Pacific.

Los arrecifes de coral se caracterizan por ser uno de los ecosistemas marinos taxonómicamente más diversos y por poseer altas tasas de productividad primaria (VásquezDomínguez 2003). Estos sistemas proveen un hábitat para un sinnúmero de especies sirviendo de refugio, como zona de alimentación, crecimiento y reproducción; además poseen la función de mantener la interacción entre la zona costera y oceánica (Nybakken 1993). Los arrecifes son reconocidos actualmente como ecosistemas complejos, con múltiples estados estables fluctuantes en el tiempo (Connell et al. 1997, Jackson y Johnson 2000, Edmunds 2002) En los últimos 20-50 años, dichas fluctuaciones han culminando en el dramático incremento de la mortalidad coralina y la degradación del arrecife (Jackson 1997, 2001, Wood 2001, Pandolfi 2003). Las causas de esta crisis son una compleja mezcla del disturbio directo de actividades antropogénicas y el estrés ocasionado por cambios climáticos que afectan directamente la distribución espacio-temporal de la comunidad arrecifal. Algunas de estas 
causas son los cambios en la temperatura y salinidad del agua, altos niveles de irradiancia, transparencia del agua, la fuerza hidrodinámica y la aportación de sedimentos (Done 1982, Anthony et al. 2007). Particularmente, el aporte de sedimento no asimilable sobre la superficie de la colonia coralina afecta la reproducción e inhibe el reclutamiento larval (Rogers 1990, Hunte y Wittenberg 1992, Wittenberg y Hunte 1992), interfiere con la ecología y estructura de la comunidad de corales pétreos (Sttaford-Smith 1993, OchoaLópez et al. 1998), reduce la capacidad de respiración, captura de alimento y fotosíntesis (Szmant-Froelich et al. 1981, Abdul-Salem y Porter 1988, Stafford-Smith y Ormond 1992), incrementa la demanda de energía para la remoción activa del sedimento (Stafford-Smith y Ormond 1992, Riegl y Branch 1995), daña los pólipos en sentido mecánico (Rogers 1990) y bloquea el intercambio agua-tejido del coral afectando el metabolismo del par simbionte (Jordán-Dahlgren 1989). Además, la sedimentación afecta también al resto de la comunidad béntica, reduciendo el espacio disponible para el forrajeo y el movimiento, y altera la topografía del sustrato (Rogers 1990). Dada su importancia, los estudios respecto a la sedimentación y sus efectos en las comunidades coralinas son numerosos en el Indo Pacífico (Mapstone et al. 1989, Stafford-Smith 1993, Fabricius y Wolanski 2000) y en el Caribe (Hunte y Wittenberg 1992, Whittenberg y Hunte 1992), pero escasos en el Pacífico Oriental (Cortés y Risk 1985). En México, excepto por el estudio de Ochoa-López et al. (1998) en Islas Revillagigedo, no existen informes del aporte de sedimentos hacia las comunidades coralinas. En este sentido, el presente trabajo pretende evaluar la tasa de sedimentación y su variación espacio-temporal en seis localidades de Bahías de Huatulco, Oaxaca en el Pacífico mexicano.

\section{MATERIAL Y MÉTODO}

Área de estudio: El presente estudio se realizó en seis comunidades arrecifales de Bahías de Huatulco, Oaxaca, situadas en la costa oriental de México: Isla San Agustín $\left(15^{\circ} 41^{\prime} \mathrm{N}, 96^{\circ} 14^{\prime} \mathrm{W}\right)$, Jicaral-Chachacual $\left(15^{\circ} 42^{\prime} \mathrm{N}, 96^{\circ} 13^{\prime} \mathrm{W}\right)$, Dos Hermanas $\left(15^{\circ} 42^{\prime}\right.$ N, 96 $\left.12^{\prime} \mathrm{W}\right)$, Isla Cacaluta $\left(15^{\circ} 43^{\prime} \mathrm{N}, 96^{\circ} 09^{\prime}\right.$ W), La Entrega $\left(15^{\circ} 44^{\prime} \mathrm{N}, 96^{\circ} 07^{\prime} \mathrm{W}\right)$ e Isla Montosa $\left(15^{\circ} 45^{\prime} \mathrm{N}, 96^{\circ} 04^{\prime} \mathrm{W}\right)$.

El clima que presenta la región corresponde al cálido subhúmedo con lluvias abundantes en verano y escasas lluvias invernales, con una temperatura media anual de $26.9{ }^{\circ} \mathrm{C}$ (García 1973, de Alba y Reyes 1998). El régimen pluvial es de tipo torrencial presentando una precipitación media anual entre 800 y $1500 \mathrm{~mm}$, y un porcentaje invernal menor al 5\% (García 1973, Conanp 2003). Debido a que la precipitación se concentra entre junio a octubre, y a que existe escasa retención de humedad en el suelo, la zona carece de corrientes permanentes, excepto el Río Copalita, por lo que los procesos originados en las desembocaduras en la costa son insignificantes ya que son corrientes pequeñas e intermitentes (de la Lanza-Espino 1991).

Con respecto a las condiciones oceanográficas, el agua tropical superficial (altas temperaturas y baja salinidad) baña regularmente la costa de Oaxaca; mientras que en condiciones invernales extremas el agua superficial de la Corriente de California (fría y de baja salinidad) alcanza el Golfo de Tehuantepec (Fiedler 1992). Por tal razón, durante el invierno las características oceanográficas de la costa de Oaxaca están determinadas por eventos locales de viento, calentamiento solar y lluvia (Trasviña 1991) y las únicas corrientes apreciables son de este origen. Mientras que durante el verano, las condiciones tropicales de la Corriente Costera de Costa Rica son el aporte principal de las condiciones oceánicas hacia esta región (Trasviña 1991, Fiedler 1992).

Los registros de la temperatura del agua fluctúan entre 20.5 y $34.4^{\circ} \mathrm{C}$, y los de salinidad entre 17.3 y $40.1 \mathrm{ppm}$ con una media de 34.6 ppm (Anónimo 1978). La profundidad media de la termoclina es de $60 \mathrm{~m}$ durante la mayor parte del año, misma que se incrementa hacia el sur a partir del Golfo de Tehuantepec (Cromwell 1958, Secretaría de Marina 1978, Fiedler 1992). 
Sin excepción, las zonas arrecifales de Huatulco se presentan en bahías o detrás de islas y rocas protegidas del oleaje directo. Dichas comunidades se caracterizan por la presencia de 12 especies hermatípicas correspondientes a cuatro géneros (Psammocora, Pocillopora, Porites, Pavona), siendo dominantes los pocilloporidos: Pocillopora verrucosa (Ellis y Solander 1786), Pocillopora capitata Verrill, 1864 y Pocillopora damicornis (Linnaeus 1758). Cabe destacar que la mayor parte de las comunidades poseen una cobertura de coral vivo de alta (30-50\%) a muy alta (6090\%) (Glynn y Leyte-Morales 1997).

Obtención de muestras: Durante febrero 2006 y enero 2007 (exceptuando abril, julio y agosto), fueron realizados muestreos de sedimentos en las comunidades arrecifales referidas. Dos estructuras por localidad, fueron fijadas al sustrato a una profundidad de $\sim 2-10$ $\mathrm{m}$ mediante varillas de acero de $1.2 \mathrm{~m}$. Las estructuras consisten de rejas de plástico con 12 compartimentos (similares a las utilizadas para almacenar botellas de refresco) y en sólo cuatro de los compartimentos, se sujetaron mediante cinchos plásticos, recolectores de sedimento (tubos de PVC de $21 \mathrm{~cm}$ de alto, diámetro de $4.4 \mathrm{~cm}$ ) de manera vertical. Los recolectores de sedimento fueron retirados con una periodicidad regular de $\sim 38$ días, y fueron reemplazados por otros. Los sedimentos fueron procesados mediante la técnica sugerida por Rogers (1994). Una vez en el laboratorio, el sedimento fue despojado de partículas orgánicas que representaran una sobreestimación del peso del mismo (e.g. organismos grandes) y lavado profusamente con agua destilada con el fin de eliminar el contenido de sales de la muestra. Posteriormente, las muestras fueron filtradas mediante papel Whatman \#41 (previamente pesado) y secadas en un horno a $70^{\circ} \mathrm{C}$ hasta alcanzar un peso constante $(\sim 24-72$ horas). Finalmente, se obtuvo el peso neto del sedimento utilizando una balanza analítica y restando el peso del papel filtro al mismo. Los datos obtenidos del sedimento procesado fueron utilizados para la determinación de la tasa de sedimentación ( $\mathrm{mg} \mathrm{cm} \mathrm{cí}^{-1}$ ), considerando el número de días que los recolectores de sedimento estuvieron sumergidos y el diámetro de la abertura de éstos.

Análisis de datos: A partir de los datos de sedimentación se realizó un análisis de varianza (ANDEVA) de 1 vía para determinar si existen diferencias significativas entre la cantidad de sedimento depositado en las comunidades estudiadas, así como la variación de la sedimentación respecto al tiempo. Posteriormente se llevó a cabo una prueba a posteriori de Tukey para muestras desiguales para detectar aquellas localidades y tiempos entre los que ocurrían diferencias. Finalmente se llevó a cabo una correlación de Spearman para determinar la posible relación entre la precipitación pluvial registrada en la región y la tasa de sedimentación (Zar 1999). El nivel de significancia para todos los análisis fue de $\alpha=0.05$.

\section{RESULTADOS}

La tasa de sedimentación es altamente heterogénea en la región $\left(F_{5,36}=7.06, P<0.01\right)$; los valores más altos se presentan en Isla Montosa $\left(653.31 \mathrm{~kg} \mathrm{~m}^{-2}\right.$ año $\left.{ }^{-1}\right)$ e Isla Cacaluta $(450.09 \mathrm{~kg}$ $\mathrm{m}^{-2}$ año $\left.{ }^{-1}\right)$, intermedios en San Agustín (133.30 $\mathrm{kg} \mathrm{m}^{-2}$ año $\left.{ }^{-1}\right)$, Jicaral-Chachacual (155.18 kg $\mathrm{m}^{-2}$ año $\left.^{-1}\right)$ y Dos Hermanas $\left(92.53 \mathrm{~kg} \mathrm{~m}^{-2} \mathrm{año}^{-1}\right)$ y bajos en La Entrega (14.33 $\mathrm{kg} \mathrm{m}^{-2}$ año ${ }^{-1}$ ) (Fig. 1). La prueba de Tukey sugiere que de acuerdo a la cantidad de sedimento, las comunidades pertenecientes a Isla Cacaluta e Isla Montosa son iguales entre sí, pero significativamente diferentes al grupo formado por JicaralChachacual, Dos Hermanas, San Agustín y La Entrega, quienes presentan niveles de sedimentación homogéneos.

Por otra parte, la sedimentación registrada en Bahías de Huatulco no varía significativamente a lo largo del año $\left(F_{7,34}=0.85, P>\right.$ $0.5)$, lo que sugiere que el patrón de variación entre niveles de sedimentación es más importante entre localidades que a través del tiempo. Sin embargo, es evidente que durante lluvias la tasa de sedimentación asciende de 

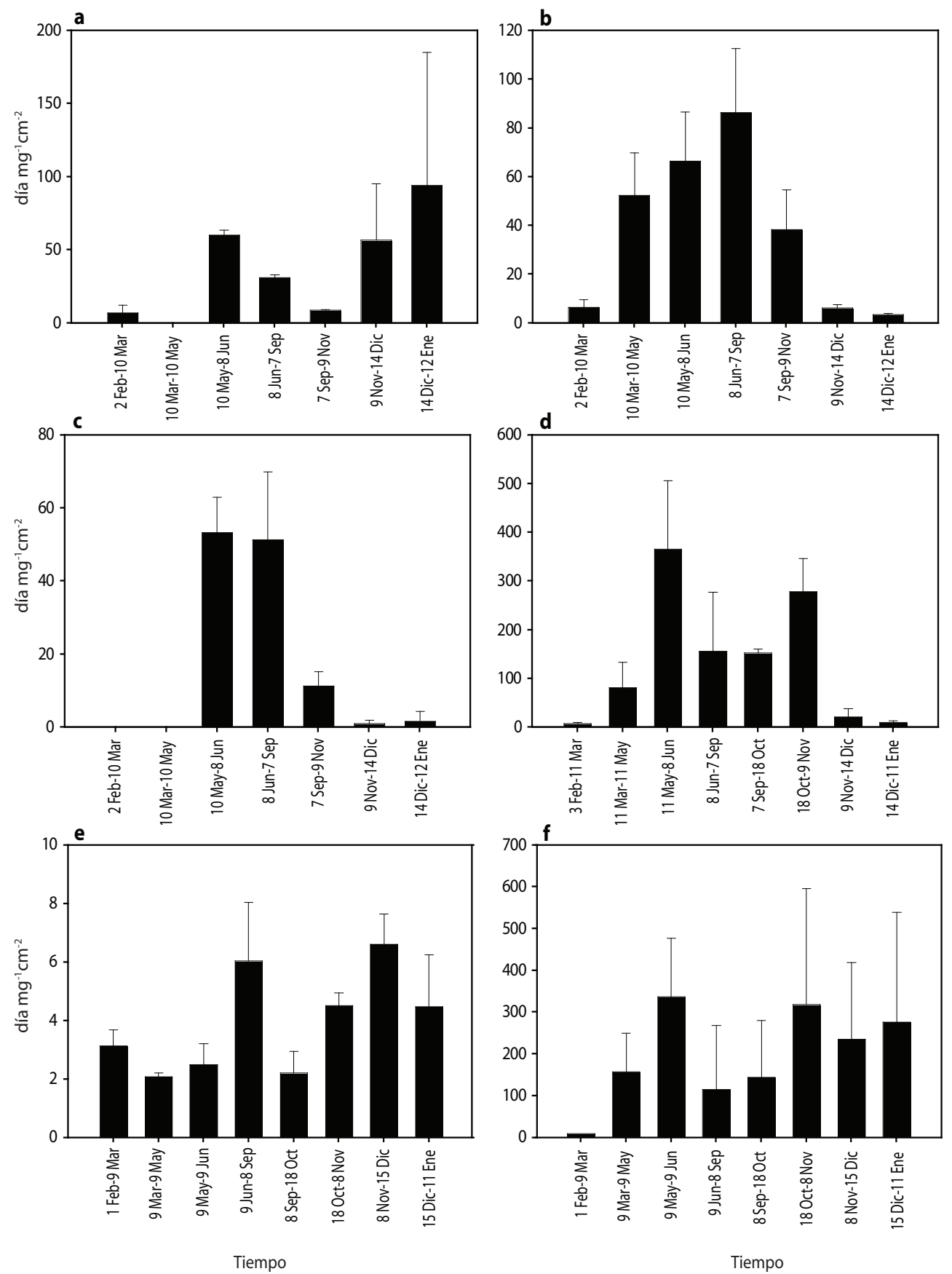

Fig. 1. Tasa de sedimentación ( $\mathrm{mg} \mathrm{cm}^{-2} \mathrm{día}^{-1} \pm \mathrm{DE}$ ) durante un ciclo anual (febrero 2006-enero 2007) en el área de Bahías de Huatulco. $\mathrm{a}=$ San Agustín, $\mathrm{b}=$ Jicaral-Chachacual, $\mathrm{c}=$ Dos Hermanas, $\mathrm{d}=$ Isla Cacaluta, e $=$ La Entrega, $\mathrm{f}=$ Isla Montosa. Note las diferentes escalas de los ejes verticales.

Fig. 1. Sedimentation rate $\left(\mathrm{mg} \mathrm{cm}^{-2}\right.$ day $\left.^{-1} \pm \mathrm{SD}\right)$ during an annual cycle (February 2006-January 2007) at the Bahías de Huatulco area. $\mathrm{a}=$ San Agustín, $\mathrm{b}=$ Jicaral-Chachacual, $\mathrm{c}=$ Dos Hermanas, $\mathrm{d}=$ Isla Cacaluta, e $=\mathrm{La}$ Entrega, $\mathrm{f}=\mathrm{Isla}$ Montosa. Note different scales at vertical axes. 
manera considerable. Durante temporada de secas (noviembre-marzo) el área de Bahías de Huatulco recibe entre $6.8-73.5 \mathrm{mg} \mathrm{cm}^{-2}$ día $^{-1}$, mientras que durante la temporada de lluvias (mayo-octubre) la cantidad de sedimento se incrementa a 74.5-147.6 mg cm $\mathrm{día}^{-1}$, es decir, durante la temporada de lluvias la cantidad de sedimento es 141-1088\% mayor que durante secas (Mann-Whitney $U=137, n=42, P=$ $0.03)$. Los análisis de correlación entre la cantidad de sedimento registrada en las localidades y la precipitación pluvial media en la región evidenciaron que sólo Isla Cacaluta se encuentra significativamente relacionada a dicho factor (Spearman $R=0.83, n=8, P=0.011$ ); $\sin$ embargo, la tasa de sedimentación media de Bahías de Huatulco despliega el mismo patrón (Spearman $R=0.84, n=8, P=0.009$ ), sugiriendo que el sedimento depositado en el área de estudio es relativamente dependiente de la escorrentía regional (Fig. 2).

\section{DISCUSIÓN}

Los resultados muestran que la tasa de sedimentación entre localidades es heterogénea, pero que a nivel regional está estrechamente relacionado a la precipitación pluvial de la zona. Entre localidades, Isla Montosa e Isla Cacaluta experimentaron niveles de sedimentación $300 \%$ más altos que San Agustín, Jicaral-Chachacual, Dos Hermanas y La Entrega. Aunque carecemos de elementos inequívocos para explicar las diferencias, muy probablemente éstas estén relacionadas a las condiciones hidrodinámicas imperantes en las localidades. Mientras las comunidades arrecifales de San Agustín, Jicaral-Chachacual, Dos Hermanas y La Entrega se desarrollan en bahías protegidas del oleaje directo, en Isla Cacaluta e Isla Montosa las comunidades están expuestas a una alta energía del oleaje y a la corriente que atraviesa el canal que conecta la

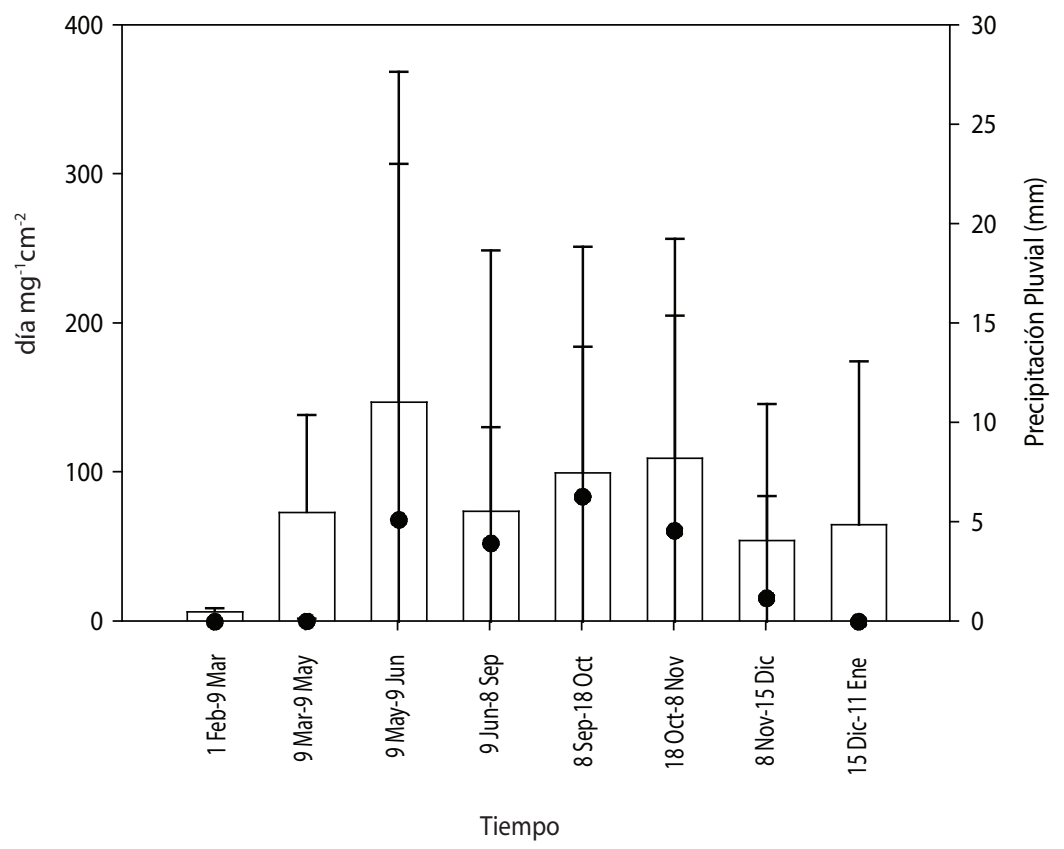

Fig. 2. Tasa de sedimentación $\left(\mathrm{mg} \mathrm{cm}^{-2} \mathrm{día}^{-1}\right)$ y precipitación pluvial media $(\mathrm{mm})$ en Bahías de Huatulco durante un ciclo anual (febrero 2006-enero 2007). Barras = tasa de sedimentación, círculos = precipitación pluvial media.

Fig. 2. Sedimentation rate $\left(\mathrm{mg} \mathrm{cm} \mathrm{cm}^{-2}\right.$ day $\left.^{-1}\right)$ and mean precipitation $(\mathrm{mm})$ at Bahías de Huatulco during an annual cycle (February 2006-January 2007). Bars $=$ Sedimentation rate, dots $=$ mean precipitation. 
isla al continente. En particular, las condiciones hidrodinámicas imperantes en las islas facilitan la resuspensión (e.g., oleaje) y el transporte (e.g., corrientes) de sedimentos de otras fuentes (Rogers 1990). Un elemento adicional es la relativa cercanía de Isla Montosa al Río Copalita quien durante temporada de lluvias extiende su pluma sedimentaria más allá de Isla Cacaluta (Obs. personal).

La tasa de sedimentación registrada en las comunidades arrecifales de Bahías de Huatulco está en función de la cantidad de agua fresca descargada por el Río Copalita y en menor medida por el Río Coyula, el volumen y la intensidad de la precipitación pluvial, y de la relación entre éstas con las condiciones de los suelos y del uso del suelo que se desarrolla en las cuencas (Hubbard 1987). No existen indicios de que previo al desarrollo del complejo turístico de la zona, la sedimentación asociada a la descarga de los ríos así como a la precipitación pluvial tuviera efectos letales en las comunidades coralinas. Sin embargo, luego de su expropiación por el gobierno federal en 1984, Bahías de Huatulco pasó de ser una zona agrícola y pesquera con una densidad humana baja, para convertirse en un desarrollo turístico cuya construcción implicó la apertura de áreas de selva, la remoción de grandes cantidades de suelos y la modificación de afluentes naturales (Conanp 2003). Aunque nunca cuantificado, la cantidad de sedimento vertido al mar, así como sus efectos en las comunidades arrecifales debió ser enorme. Existen evidencias de que coincidente con la construcción del complejo turístico desaparecieron arrecifes que se desarrollaban en La Mina, La Dársena y Manzanilla, además de una porción importante de La Entrega (Glynn y Leyte-Morales 1997, Leyte-Morales 2000, López-Pérez et al. 2002) considerado el más diverso y extenso con que cuenta la región, sin que a la fecha existan signos de recuperación.

La tasa de sedimentación media registrada en arrecifes no expuestos a la actividad humana oscila entre $1-10 \mathrm{mg} \mathrm{cm}^{-2} \mathrm{día}^{-1}$ (Rogers 1990), por lo que los niveles de sedimentación registrados en Bahías de Huatulco podrían considerarse potencialmente crónicos. En arrecifes costeros de Australia se han registrado concentraciones de material particulado de 5-40 $\mathrm{mg} \mathrm{l}^{-1}$ (Fabricious y Wolanski 2000, Larcombe et al. 2001), 1.97-7.32 $\mathrm{mg} \mathrm{l}^{-1}$ en Barbados (Wittenberg y Hunte 1992) y 0.5-1.1 $\mathrm{mg} \mathrm{cm}$ día $^{-1}$ en Jamaica (Dodge et al. 1974), todas ellas consideradas como no alarmantes por no ser subletales o letales; mientras que tasas de sedimentación mayores a $200 \mathrm{mg}$ $\mathrm{cm}^{-2}$ día $^{-1}$ son raras y potencialmente deletéreas, y han sido registradas para arrecifes del Pacífico Centro Americano (Cortés y Risk 1985) y Australia (Mapstone et al. 1989) para periodos de días a semanas. Carecemos de información respecto al grado de afectación que los niveles de sedimentación registrados causan en los arrecifes de Bahías de Huatulco, no obstante, los valores observados son elevados en términos de la capacidad de rechazo y de la tolerancia al sedimento. Experimentos de laboratorio y campo evidencían que los niveles subletales y letales de estrés asociados a la sedimentación varía entre especies y localidades en función de la tolerancia, capacidad de rechazo activo y pasivo de sedimento, tipo de sedimento, tamaño y disposición de la colonia (determinantes para la remoción activa), además del posible incremento en la tolerancia fisiológica a los sedimentos por parte de los corales pétreos de la zona (Bak y Elgershivzen 1976, Rogers 1990, StaffordSmith 1993, Fabricious y Wolanski 2000, Anthony et al. 2007, Fabricius et al. 2007).

Stafford-Smith (1993) indica que la supervivencia de corales en condiciones de sedimentación por arriba de $200 \mathrm{mg} \mathrm{cm}{ }^{-2}$ día $^{-1}$ sólo se presenta mediante una tasa de rechazo de sedimentos alta vía modificaciones morfológicas, el desarrollo de arrecifes en zonas turbulentas, una alta inversión energética durante el rechazo activo de sedimento, así como el incremento en la tolerancia fisiológica al sedimento. En este sentido, la cantidad de sedimento registrado en Bahías de Huatulco durante el periodo de lluvias debe ejercer un nivel de estrés considerable en las comunidades, particularmente en Isla Montosa e Isla Cacaluta donde los niveles 
de sedimentación alcanzan $366 \mathrm{mg} \mathrm{cm}^{-2}$ día $^{-1}$. No obstante, el nivel de desarrollo coralino alcanzado en Bahías de Huatulco (Glynn y Leyte-Morales 1997), así como la evidencia de reproducción (Rodríguez-Troncoso 2004, 2006) y reclutamiento sexual (López-Pérez et al. 2007), permite sugerir que el posible efecto subletal y letal de los niveles de sedimentación de la zona, es potencialmente reducido por la eficiencia de la remoción activa de sedimento por parte de las especies de coral y la influencia pasiva de la turbulencia del agua en la remoción del sedimento.

Es importante subrayar, sin embargo, que el efecto combinado de la sedimentación natural, aunado a una constante y alta sedimentación como resultado de actividades antropogénicas debido al mal manejo de las cuencas, afecta significativa y negativamente los arrecifes, en ocasiones con consecuencias letales. Debido a lo anterior, se sugiere que los planes de desarrollo de Bahías de Huatulco deben considerar que la ineficiencia en el manejo turístico y la deforestación o urbanización de la cuenca, incrementaría aún más la ya de por si alta carga sedimentaria de la zona, lo que podría promover un cambio en la estructura arrecifal y la eventual pérdida de las comunidades coralinas.

\section{AGRADECIMIENTOS}

Agradecemos al Parque Nacional Huatulco por las facilidades y el permiso para trabajar en el área. El trabajo se realizó mediante apoyo del proyecto "Diagnóstico de los recursos naturales de la Bahía y micro-cuenca del Río Cacaluta" financiado por el fondo SemarnatCONACYT (registro 0605). El apoyo de Eladio Espindola, Andrés Pacheco, Imelda López, Ariadna López, Trinidad Domínguez, Francisco Benítez y Raúl (suegro) Granja fue invaluable durante el trabajo de campo. El artículo es una colaboración del Grupo "Arrecifes del Pacífico" de la Red Mexicana de Investigación Ecológica a largo Plazo (Red Mex-LTER). Héctor Reyes (UABCS, La Paz) y Pedro Medina (U de G, Vallarta) revisaron el manuscrito e hicieron sugerencias para incrementar la calidad del mismo.

\section{RESUMEN}

Aún cuando la sedimentación en arrecifes es importante pues sus efectos alteran procesos como la simbiosis, reproducción, reclutamiento y crecimiento, se carece de estudios en el Pacífico mexicano. Debido a lo anterior, se evaluó la tasa de sedimentación y su variación espacio-temporal en seis comunidades coralinas de Oaxaca. Durante febrero 2006- enero 2007 (exceptuando abril, julio y agosto), instalamos al azar dos estructuras con cuatro recolectores de sedimento cada una. Los recolectores fueron reemplazados aproximadamente cada 38 días y los sedimentos fueron enjuagados, filtrados, secados y pesados. La tasa de sedimentación fue heterogénea entre localidades $\left(F_{5,36}=7.06, P<0.01\right)$; fue alta en Isla Montosa (653.31 $\mathrm{kg} \mathrm{m}^{-2}$ año ${ }^{-1}$ ) e Isla Cacaluta (450.09 $\mathrm{kg} \mathrm{m}^{-2}$ año ${ }^{-1}$ ), intermedia en San Agustín, Jicaral-Chachacual y Dos Hermanas (155.18-92.53 $\mathrm{kg} \mathrm{m}^{-2}$ año $^{-1}$ ) y baja en La Entrega (14.33 kg $\mathrm{m}^{-2}$ año-1 $)$. La tasa de sedimentación no varió a lo largo del año $\left(F_{7,34}=0.85, P>0.5\right)$; no obstante, durante la temporada de secas (noviembre-marzo) la tasa de sedimentación en la zona osciló entre $6.8-73.5 \mathrm{mg} \mathrm{cm}^{-2} \mathrm{día}^{-1}$, mientras que durante lluvias (mayo-octubre) osciló entre 74.5-147.6 mg $\mathrm{cm}^{-2}$ día $^{-1}$, es decir, durante la temporada de lluvias la tasa de sedimentación fue 141-1088\% mayor que durante secas (Mann-Whitney $U=137, n=42, P=0.03$ ). La relación entre la cantidad de sedimento y la precipitación pluvial fue significativa (Spearman $R=0.83, n=8, P=0.009$ ), sugiriendo que el aporte de sedimento está relacionado con la escorrentía regional. La tasa de sedimentación registrada en Isla Montosa (366.64 mg cm día $^{-1}$ ) e Isla Cacaluta (366.03 $\mathrm{mg} \mathrm{cm}^{-2}$ día $^{-1}$ ) durante la temporada de lluvias, puede ser considerada como letal-subletal en función de la tolerancia y de la capacidad de rechazo de sedimento por parte de los corales; no obstante, la ausencia de mortalidad coralina, en respuesta a los niveles de sedimentación registrados durante la época de lluvias, y el desarrollo coralino observado en la región pudiera estar relacionado al efecto combinado de: a) la eficiencia de la remoción activa de sedimento, b) el posible incremento en la tolerancia fisiológica al sedimento por parte de los corales pétreos de Bahías de Huatulco, y c) la remoción pasiva de sedimentos vía la turbulencia de la zona. No obstante, el efecto combinado de la sedimentación natural aunado a la sedimentación resultado de actividades antropogénicas en la región, podría promover un cambio en la estructura arrecifal provocando la eventual pérdida de las comunidades coralinas en Bahías de Huatulco.

Palabras clave: sedimentos, tasa de sedimentación, comunidades coralinas, Oaxaca, Pacífico mexicano. 


\section{REFERENCIAS}

Abdul-Salem, H.A \& J.W. Porter. 1988. Physiological effects of sediment rejection on photosynthesis and respiration in three Caribbean reef corals. Proc. $6^{\text {th }}$ Int. Coral Reef Symp. 2: 285-292.

Anónimo. 1978. Temperatura y salinidad de los puertos de México en el Océano Pacífico. Secretaría de Marina/ Dirección General de Oceanografía, México. 120 p.

Anthony, K.R.N., S.R. Connolly \& O. Hoehg-Guldberg. 2007. Bleaching, energetic, and coral mortality risk: Effects of temperature, light, and sediment regime. Limnol. Oceanogr. 52: 716-726.

Bak, R.P.M \& J.H.B.M. Elgershvizen. 1976. Patterns of oil sediments rejection in corals. Mar. Biol. 37: 105-113.

Conanp. 2003. Programa de manejo Parque Nacional Huatulco. Conanp-Semarnat, México DF, México.

Connell, J.H., T.P. Hughes \& C.C. Wallace. 1997. A 30 -year study of coral abundance, recruitment, and disturbance at several scales in space and time. Ecol. Monogr. 67: 461-488.

Cortés, N.J \& M.J. Risk. 1985. A reef under siltation stress: Cahuita, Costa Rica. Bull. Mar. Sci. 36: 339-356.

Cromwell, T. 1958. Termocline topography, horizontal currents and ridging in the eastern tropical Pacific. Inter-am. Trop. Tuna Com. Bull. 3: 135-164.

de Alba E, Reyes ME. 1998. Parte I. El País: Contexto físico, p. 1-35. In J. Sarukan-Kermez (ed.). La diversidad biológica de México: estudio de país, 1998. CONABIO, México DF, México.

de la Lanza-Espino, G. 1991. Oceanografía de mares mexicanos. AGT, México. 518 p.

Dodge, R.E., R.C. Aller \& J. Thompson. 1974. Coral growth related to resuspension of bottom sediments. Nature 247: 574-577

Done, T.J. 1982. Patterns in the distribution of coral communities across the central Great Barrier Reef. Coral Reefs 1: 95-107.

Edmunds, P.J. 2002. Long-term dynamics of coral reefs in St. John, US Virgin Islands. Coral Reefs 21: 357367.

Fabricius, K.E \& E. Wolanski. 2000. Rapid smothering of coral reef organism by muddy marine snow. Estuar. Coast. Shelf Sci. 50: 115-120.
Fabricius, K.E., Y. Golbuu \& S. Victor. 2007. Selective mortality in coastal reef organisms from an acute sedimentation event. Coral Reefs 26: 69.

Fiedler, P.C. 1992. Seasonal climatologies and variability of eastern tropical Pacific surface waters. NOAA Tech. Rep. NMFS. 109: 1-65.

García, E. 1973. Modificaciones al sistema de clasificación climática de Kôpen para adaptarla a las condiciones de la República Mexicana. Inst. Geogr. UNAM, México.

Glynn, P.W \& G.E. Leyte-Morales. 1997. Coral reefs of Huatulco, West México: Reef development in upwelling Gulf of Tehuantepec. Rev. Biol. Trop. 45: 1033-1047.

Hubbard, D.K. 1987. A general review of sedimentation as it relates to environmental stress in the Virgin Islands Biosphere Reserve and the Eastern Caribbean in general. Biosphere reserve research report no. 20. National Park Service. Islas Vírgenes, EEUU.

Hunte, W \& M. Wittenberg. 1992. Effects of eutrophication and sedimentation on juvenile corals. II. Settlement. Mar. Biol. 114: 625-631.

Jackson, J.B.C. 1997. Reefs since Columbus. Coral Reefs 16: S23-S32.

Jackson, J.B.C \& K.G. Johnson. 2000. Life in the last few million years. Paleobiology (Supl.) 26: 221-235.

Jackson, J.B.C. 2001. What was natural in the coastal oceans?. Proc. Natl. Acad. Sci. USA 98: 5411-5418.

Jordán-Dahlgren, E. 1989. Efecto de la morfología del sustrato en el desarrollo de la comunidad coralina. An. Inst. Cienc. del Mar y Limnol. Univ. Nal. Auton. México 16: 105-117

Larcombe, P., A. Costen \& K.J. Woolfe. 2001. The hydrodynamic and sedimentary setting of nearshore coral reefs, central Great Barrier Reef Shelf, Australia: Paluma Shoals, a case study. Sedimentology 48: 811-835.

Leyte-Morales, G.E. 2000. Perturbaciones naturales y antropogénicas en las comunidades coralinas de Oaxaca (1977-1998). Resumen. XII Congreso Nacional de Oceanografía. Huatulco, Oaxaca, México.

López-Pérez, R.A., L.M. Hernández-Ballesteros \& T. Herrera-Escalante. 2002. Cambio en la dominancia de la comunidad arrecifal en Chachacual, Bahías de Huatulco, Oaxaca. Ciencia y Mar 16: 33-38. 
López-Pérez, R.A., M.G. Mora-Pérez \& G.E. LeyteMorales. 2007. Coral recruitment at Bahías de Huatulco, western México: Implications for coral community structure and dynamics. Pac. Sci. 61: 355-369

Mapstone, B.D., J.H. Choat, R.L. Cumming \& W.G. Oxley. 1989. The fringing reefs of Magnetic Island: benthic biota and sedimentation; a baseline survey. Great Barrier Reef Marine Park Authority Townsville, Queensland, Australia.

Newman, M.J.H., G. Paredes, R.R. Warner \& J.B.C. Jackson. 2003. Global trajectories of the long-term decline of coral reef ecosystems. Science 301: 955-958.

Nybakken, J.W. 1993. Marine biology: an ecological approach. Harper, Nueva York, EEUU.

Ochoa-López, E., H. Reyes-Bonilla \& J. Ketchum-Mejía. 1998. Daños por sedimentación a las comunidades coralinas del sur de la Isla Socorro, Archipiélago de Revillagigedo, México. Cien. Mar. 24: 233-240.

Pandolfi, J.M., R.H. Bradbury, E. Sala, T.P. Hughes, K.A. Bjorndal, R.G. Cooke, D. McArdle, L. McClenachan, M.J.H. Newman, G. Paredes, R.R. Warner, J.B.C. 2003. Global trajectories of the long-term decline of coral reef ecosystems. Science 301: 955-958.

Riegl, B \& G.M. Branch. 1995. Effects of sediment on the energy budgets of four scleractinian (Bourne 1900) and five alcyonacean (Lamouroux 1816) corals. J. Exp. Mar. Biol. Ecol. 186: 259-275.

Rogers, C.S. 1990. Responses of coral reefs and reef organisms to sedimentation. Mar. Ecol. Prog. Ser. 62: $185-202$

Rogers, C.S. 1994. Coral reef monitoring manual for the Caribbean and Western Atlantic. National Park Service. Islas Vírgenes, EEUU.
Secretaría de Marina. 1978. Temperatura y salinidad de los puertos de México en el Océano Pacífico. Secretaría de Marina/Dirección General de Oceanografía. México.

Stafford-Smith, M.G \& R.F.G. Ormond. 1992. Sediment rejection mechanisms of 42 species of Australian scleractinian corals. Aust. J. mar. Freshwat. Res. 43: 683-705.

Stafford-Smith, M.G. 1993. Sediment-rejection efficiency of 22 species of Australian scleractinian corals. Mar. Biol. 115: 229-243.

Szmant-Froelich, A., V. Johnson, T. Hoehn, J. Battey, G.J. Smith, E. Fleischmann, J. Porter, D. Dallmeyer. 1981. The physiological effects of oil-drilling muds of the Caribbean coral Montastrea annularis. Proc. $4^{\text {th }}$ int. coral Reef Symp. 1: 163-168.

Trasviña, A. 1991. Offshore wind forcing in a coastal ocean: observations and modeling of the Gulf of Tehuantepec, México. Ph.D. Thesis, Bangor, Inglaterra.

Vásquez-Domínguez, E. 2003. Diversidad y distribución de crustáceos y equinodermos y su relación con niveles de sedimentación en arrecifes coralinos. Rev. Biol. Trop. 51: 183-194.

Wittenberg, M \& W. Hunte. 1992. Effects of eutrophication and sedimentation on juvenile corals. I. Abundance, mortality and community structure. Mar. Biol. 112: 131-138.

Wood, R. 2001. Biodiversity and the history of reefs. Geol. J. 36: 251-263.

Zar, J.H. 1999. Biostatistical analysis. Prentice, Nueva Jersey, EEUU. 
